\title{
Die Renaissance der Kronzeugenregelung ${ }^{1}$
}

\section{Einleitung}

Die Bundesregierung hat am 16.5.2007 den Entwurf eines Gesetzes zur Änderung des Strafgesetzes verabschiedet, durch welchen mit der Kronzeugenregelung eines der umstrittensten Rechtsinstitute des Strafrechts erneut und gegenüber den Vorgängerregelungen in erweiterter Form in das Strafgesetzbuch eingeführt werden soll. ${ }^{2}$ Es ist zu erwarten, dass der Bundestag noch in dieser Legislaturperiode mit der Stimmenmehrheit der großen Koalition den Entwurf verabschieden wird.

Die Figur des Kronzeugen kommt aus dem englischen Rechtsraum. Kronzeuge ist derjenige, der für die Anklage, die im Strafverfahren die Krone repräsentiert, als Belastungszeuge fungiert, obwohl er selbst der Beteiligung an den angeklagten Tatvorwürfen verdächtig oder überführt ist. Der Kronzeuge erhält für seine Kooperation als Gegenleistung eine Strafmilderung oder einen Straferlass. ${ }^{3}$

Bis 1999 galt das Kronzeugengesetz, das für die Bildung krimineller oder terroristischer Vereinigungen und damit zusammenhängende Taten die Möglichkeit eröffnete, das Verfahren einzustellen, von Strafe abzusehen oder die Strafe zu mildern. Diese zeitlich befristeten Regelungen waren unter der rot-grünen Bundesregierung nicht verlängert worden. Mehrere Initiativen des CDU/ CSU-geführten Bundesländer und der CDU/ CSU-Bundestagsfraktion fanden unter »Rot-Grün« keine Mehrheit. ${ }^{4}$ Doch bereits das geltende Strafrecht kennt für bestimmte Delikte, nämlich bei der Bildung einer kriminellen oder terroristischen Vereinigung ( $\S 129,129$ a StGB), der Geldwäsche ( $\$ 261$ StGB) und im Betäubungsmittelstrafrecht ( 31 BtMG), sog. kleine Kronzeugenregelungen. Da diese Regelungen jedoch nur einen Ausschnitt der Tatbestände abdecken, in denen der Einsatz von Kronzeugen in Betracht komme, hat sich die Bundesregierung auf die Einführung einer Kronzeugenregelung im allgemeinen Teil des StGB verständigt.

\section{Der Inhalt der geplanten Neuregelungen}

Kernstück des Gesetzentwurfes ist die Einführung eines neuen $§ 46 \mathrm{~b}$ StGB. $§ 46 \mathrm{~b}$ Abs. 1 E-StGB lautet:

1 Wichtige Hinweise verdanke ich Herrn Rechtsanwalt Georg Schulze und Herrn Assessor Carsten Stölting aus Bielefeld.

2 Vgl. Presseerklärung des Bundesjustizministeriums v. 16.5.2007 und den verabschiedeten Entwurf der Bundesregierung unter: www.bundesjustizministerium.de und bereits: Zypries, Recht und Politik 2006, 5 (6).

3 Beulke, Strafprozessrecht, 9. A. 2006, Rn. 342; Denny, ZStW 103 (1991), 269 (270).

4 Z. B. Gesetzentwürfe der CDU/ CSU-Fraktion v. 13.1.2004, BT-Drucks. 15 / 2333; BTDrucks. 14 / 5938 v. 29.4.2001. 
»Wenn der Täter einer Straftat, die mit einer im Mindestmaß erhöhten Freiheitsstrafe oder mit lebenslanger Freiheitsstrafe bedroht ist,

1. durch freiwilliges Offenbaren seines Wissens wesentlich dazu beigetragen hat, dass eine Tat nach $\S 100 a$ Abs. 2 StPO aufgedeckt werden konnte oder

2. freiwillig sein Wissen so rechtzeitig einer Dienststelle offenbart, dass eine Tat nach $\S 100$ a Abs. 2 StPO, von deren Planung er weiß, noch verhindert werden kann,

kann das Gericht die Strafe nach $§ 49$ Abs. 1 StGB mildern, wobei an die Stelle ausschließlich angedrohter lebenslanger Freiheitsstrafe eine Freiheitsstrafe nicht unter zehn Jahren tritt. Für die Einordnung als Straftat, die mit einer im Mindestmaß erhöhten Freiheitsstrafe bedroht ist, werden nur Strafschärfungen für besonders schwere Fälle und keine Milderungen berücksichtigt. War der Täter an der Tat beteiligt, muss sich sein Beitrag zur Aufklärung nach S.1 Nr. 1 über den eigenen Tatbeitrag hinaus erstrecken. Anstelle der Milderung kann das Gericht von Strafe absehen, wenn die Straftat ausschließlich mit zeitiger Freiheitsstrafe bedroht ist und der Täter keine Freiheitsstrafe von mehr als drei Jahren verwirkt hat.«

Hinsichtlich der auslegungsbedürftigen Formulierung, dass der Täter »wesentlich dazu beitragen « müsse, eine Tat aus dem Katalog aufzudecken, verweist die Begründung des Entwurfs auf die von der Rechtsprechung entwickelten Grundsätze, auf die im Rahmen der Würdigung der Neuregelungen einzugehen sein wird. ${ }^{5}$

Der in $\S 46 b$ Abs. 1 E-StGB zitierte $\S 100$ a Abs. 2 StPO muss erst noch durch das geplante Gesetz zur Neuregelung der Telekommunikationsüberwachung und anderer verdeckter Ermittlungsmaßnahmen in die StPO eingeführt werden. ${ }^{6} \mathrm{Zu}$ den Tatbeständen des zu schaffenden Katalogs zählen u.a. so unterschiedliche Tatbestände wie die $\S \S 96$ Abs. 2, 97 des Aufenthaltsgesetzes, §§ 84, 84a Asylverfahrensgesetz, Mord und Totschlag ( $\S$ 211, 212 StGB) und verschiedene Eigentums- und Vermögensdelikte wie z.B. der Bandendiebstahl ( $§ 244 \mathrm{a}$ StGB), Raub ( $\$ 249$ StGB), Erpressung ( 253 StGB) oder gewerbsmäßige Hehlerei ( $\$ 260$ StGB). Diesen Tatbeständen sei gemeinsam, dass sich die Täter häufig in »konspirativ und abgeschotteten Strukturen« bewegen oder dass es sich um schwerste Straftaten handele, die allein auf Grund ihrer besonderen Schwere eine Einbeziehung in den Anwendungsbereich rechtfertigten. ${ }^{7}$ Soweit eine Einstellung des Verfahrens in Betracht komme, könne diese nach $§ 153 \mathrm{~b}$ StPO durch die Staatsanwaltschaft mit Zustimmung des Gerichts erfolgen. ${ }^{8}$

Leitlinien für die Anwendung von $§ 46 \mathrm{~b}$ Abs. 1 StGB sollen die Absätze 2 und 3 liefern. Gem. $§ 46$ b Abs. 2 E-StGB »hat das Gericht bei seiner Entscheidung insbesondere zu berücksichtigen:

1. die Art und den Umfang der offenbarten Tatsachen und deren Bedeutung für die Aufklärung oder Verhinderung der Tat, den Zeitpunkt der Offenbarung, das Aus-

5 S. Begründung des Entwurfes zur Einführung der Kronzeugenregelung, S.11 f.

6 Der verabschiedete Entwurf der Bundesregierung vom 18.4.2007 findet sich ebenfalls unter: www.bundesjustizministerium.de.

7 S. Begründung des Entwurfes zur Einführung der Kronzeugenregelung, S.10.

8 S. Begründung des Entwurfes zur Einführung der Kronzeugenregelung, S.13. 
maß der Unterstützung der Strafverfolgungsbehörden durch den Täter und die Schwere der Tat, auf die sich seine Angaben beziehen, sowie

2. das Verhältnis der in Nummer 1 genannten Umstände zur Schwere der Straftat und der Schuld des Täters.«

Da es sich bei $\S 46 b$ StGB um eine Ermessensvorschrift handelt, kann das Gericht dem Kronzeugen die Strafmilderung wegen der Schwere seiner Schuld auch ganz verwehren. ${ }^{9}$ Die Strafmilderung findet außerdem gem. $§ 46 \mathrm{~b}$ Abs. 3 E-StGB keine Anwendung, wenn der Kronzeuge sein Wissen erst mitteilt, nachdem die Eröffnung des Hauptverfahrens gegen ihn bereits beschlossen ( $\$ 207$ StPO) ist. Dadurch solle gewährleisten werden, dass die Aussagen des Kronzeugen noch rechtzeitig auf ihre Richtigkeit überprüft werden können. Es bleibe den Gerichten allerdings unbenommen, spätere Offenbarungen gem. § 46 Abs. 2 StGB oder im Rahmen der Entscheidung über die Strafaussetzung zur Bewährung zu berücksichtigen. ${ }^{10}$

Zusätzlich will die Bundesregierung die Strafdrohungen für das Vortäuschen einer Straftat ( $\$ 145 d$ StGB) und die falsche Verdächtigung ( 164 StGB) für diejenigen Täter anheben, die durch unwahre Angaben eine Strafmilderung oder ein Absehen von Strafe erzielen wollen. $§ 145 \mathrm{~d}$ Abs. 3, 4 StGB sehen als Strafrahmen eine Freiheitsstrafe von drei Monaten bis zu fünf Jahren und in minder schweren Fällen Geldstrafe oder Freiheitsstrafe bis zu drei Jahren vor. Als Strafrahmen für falsche Verdächtigungen durch Kronzeugen bestimmt $\S 164$ Abs. 3 StGB Freiheitsstrafe von sechs Monaten bis zu zehn Jahren und in minder schweren Fällen Freiheitsstrafe von drei Monaten bis zu fünf Jahren.

Die bereits vorhandenen »kleinen Kronzeugenregelungen« sollen bis auf $\S 261$ Abs. 10 StGB, für den kein Anwendungsbereich mehr gegeben sei, neben $\S 46 \mathrm{~b}$ StGB bestehen und anwendbar bleiben, wobei § $31 \mathrm{BtMG}$ an die Neuregelung angepasst wird. Anstelle der bisherigen Milderungsmöglichkeit nach freiem Ermessen des Gerichts und der Möglichkeit von einer Bestrafung nach § 29 Abs. 1, 2, 4 oder 6 BtMG abzusehen, tritt ein Verweis auf die Strafmilderung nach $\S 49$ Abs. 1 StGB. Bei verwirkten Strafen von weniger als drei Jahren kann das Gericht auch hier von Strafe ganz absehen. Ein neu eingeführter S.2 soll klarstellen, dass auch bei § 31 BtMG die Grundsätze des § 46b Abs. 2, 3 E-StGB anzuwenden sind.

\section{Bewertung der Neuregelungen}

Über die Legitimität und den Nutzen der Kronzeugenregelung wird in der Bundesrepublik $^{11}$ bereits seit den 70er Jahren intensiv diskutiert. ${ }^{12}$ Das BVerfG hatte ihre Verfas-

9 Pressemitteilung des Bundesjustizministeriums v. 16.5.2007.

10 S. Begründung des Entwurfes zur Einführung der Kronzeugenregelung, S.15.

11 S. zu den Erfahrungen und Bewertungen im Ausland, die sich wegen unterschiedlicher Rechtstraditionen nicht vollständig auf Deutschland übertragen lassen: Denny, ZStW 103 (1991), 269 ff.; Koumbarakis, Die Kronzeugenregelung im schweizerischen Strafprozess, 2007; Jeßberger, Kooperation und Strafzumessung, 1999, S.153 ff.; Mehrens, Die Kronzeugenregelung als Instrument zur Bekämpfung organisierter Kriminalität, 2001, S.161 ff.; Paskiewicz, ZStW 114 (2002), 922 ff.; Sinner, Der Vertragsgedanke im Strafprozessrecht, 1999, S.227 ff.

12 Vgl. exemplarisch aus dem älteren Schrifttum: Jung, Straffreiheit für den Kronzeugen?, 1974; J. Meyer, ZRP 1976, 25 ff.; Middendorff, ZStW 85 (1973), 1102 ff. 
sungsmäßigkeit in seiner Entscheidung aus dem Jahr 1992 dahinstehen lassen, da die Kronzeugenregelung den Beschwerdeführer nicht beschwere. ${ }^{13}$ Trotz ihres »scheinbar punktuellen Ansatzes« berührt die Kronzeugenregelung eine große Schar eng miteinander verwobener »Grundsatzprobleme« des formellen und materiellen Strafrechts. ${ }^{14}$

\section{Bewertung aus Sicht des Kronzeugen}

Kronzeugenregelungen sind aus Sicht der Kronzeugen ambivalent. Häufig treten die Strafverfolgungsbehörden zu einem Zeitpunkt an den potentiellen Kronzeugen heran, an dem sie bereits erhebliches Wissen oder zumindest Vermutungen über dessen eigene Verstricktheit in die Taten gewonnen haben und konfrontieren ihn mit den Vorwürfen. ${ }^{15}$ Sie stellen ihn vor die Wahl, entweder zu kooperieren - was einen nachhaltigen Einfluss auf das weitere Strafverfahren habe - oder anderenfalls dafür zu sorgen, dass auch er mit einer langjährigen Haftstrafe zu rechnen habe. Mag es sich hierbei auch um keinen Zwang zur Aussage im Sinne von § 136a StPO handeln, so wird die Kooperation aus Sicht des Kronzeugen doch zu einer Art Obliegenheit, die seine freie Entschließung erheblich tangiert. ${ }^{16}$ Dem Angesprochenen wird die Möglichkeit eines Vorteils versprochen, wenn er sich zu Tatvorwürfen äußert und vor allem weitere Personen beschuldigt. Dabei besteht allerdings auch die Gefahr, sich durch das Offenbaren bislang unbekannter Taten erheblich selbst zu belasten. Über die vielfältigen Risiken seiner Mitwirkung wird der Kronzeuge in der Praxis häufig nicht (hinreichend) aufgeklärt. ${ }^{17}$ Eine umfassende Beratung durch die Verteidigung setzt in diesem Fall die Kenntnis des gesamten Ermittlungsergebnisses zum Zeitpunkt der Beratung voraus. ${ }^{18}$ Zum Zeitpunkt der Aussage des Kronzeugen sind die Ermittlungen jedoch noch im vollen Gange, so dass der Verteidigung gem. § 147 Abs. 2 StPO die Akteneinsicht unter Verweis auf die Gefährdung des Untersuchungszwecks versagt werden kann. Eine solche Verweigerung des Akteneinsichtsrechts dürfte häufig der Fall sein, da es gerade zur Ermittlungstaktik gehört, den potentiellen Kronzeugen über den Informationsstand der Staatsanwaltschaft im Unklaren zu belassen, um ihn zu »umfassenden Aussagen « zu zwingen. Da ein Rechtsanspruch auf eine entsprechende Strafmilderung nicht besteht, muss der Kronzeuge in Vorleistung treten, ohne Gewissheit zu haben, tatsächlich in den Genuss der Strafmilderung zu kommen. ${ }^{19}$ Er kann sich vor allem

13 BVerfG, NJW 1993, $190 \mathrm{f}$.

14 Jung, Straffreiheit für den Kronzeugen?, S.100.

15 Gössner, Neue Kriminalpolitik 2 / 2000, 5 (6); Ransiek, StV 1996, 446 (449).

16 Vgl. hierzu auch: Bocker, Der Kronzeuge, 1991, S.90; Endriß, StraFo 2004, 151, 153 f.; Kempf, StV 1999, 67 (68): »Erpressung «. Die Schwelle zu § 136a StPO ist freilich überschritten, wenn dem potentiellen Kronzeugen absichtlich schwerere Taten vorgeworfen werden als die Verdachtslage zulässt, um ihn zur Kooperation zu veranlassen, s. Krauß, StV 1989, 315 (322).

17 Endriß, StraFo 2004, 151 (154).

18 Endriß, StraFo 2004, 151 (154); Stellungnahme des Strafrechtsausschusses des DAV, StV 2001, 317 (319).

19 Erdem, Forum Recht 2007, 64 (65). Laut Mühlhoff / Mehrens, Das Kronzeugengesetz im Urteil der Praxis, 1999, S.98, dürfte dies ein Grund für die geringe praktische Relevanz des Kronzeugengesetzes gewesen sein. Solche Bedenken äußern bereits Hassemer, StV 1986, 550 (551); Kunert / Bernsmann, NStZ 1989, 449 (457); Scheerer, StV 1989, 72 (83). 
nicht sicher sein, ob ihm nicht bereits ein anderer Beschuldigter zuvorgekommen ist, was eine Anwendung der Kronzeugenregelung ebenfalls ausschließen kann. ${ }^{20}$

Bei Zweifeln an der Richtigkeit der Entscheidung fällt ein »Rückzug« ausgesprochen schwer, da er durch die Kooperation seine Verteidigungsposition aufgegeben hat und befürchten muss, dass ein nachträgliches Scheitern der Kooperation für ihn mit nachteiligen Folgen bei der Strafzumessung verbunden ist. ${ }^{21}$

Kronzeugen begeben sich zudem durch ihre Aussagen nicht selten in enorme Gefahr $^{22}$ und müssen mit der Ablehnung ihres gesamten bisherigen sozialen Umfelds rechnen. Dem Gesetzesentwurf sind zur Problematik des wirksamen Schutzes des Kronzeugens jedoch keine Ausführungen zu entnehmen. ${ }^{23}$

Bei alldem verwundert es nicht, dass potentielle Kronzeugen von der Kronzeugenregelung bislang nur zurückhaltend Gebrauch gemacht haben.

\section{Beweisrechtliche Problematik}

Die Kronzeugenregelung ist aber vor allem aus Sicht einer der Gerechtigkeit verpflichteten Strafrechtspflege und aus Sicht der von den Kronzeugen Belasteten mit gravierenden Problemen konfrontiert. Eine der zentralen Schwierigkeiten der Kronzeugenregelung ist beweisrechtlicher Natur. Die Kronzeugenregelung kann nur dann ein legitimes Instrument der Strafverfolgung darstellen, wenn sie einen Beitrag zur Aufklärung der Wahrheit leisten kann. ${ }^{24}$ Gerade der Beweiswert der Aussagen von Kronzeugen ist jedoch häufig mehr als fraglich. Mit Recht stellt Karl Peters fest: »Falschaussagen sind geradezu vorprogrammiert, wenn der Vernommene sich Vorteile von der Aussage verspricht ... « ${ }^{25}$ Es besteht die gegenüber einem unberechtigten Freispruch um ein Vielfaches unerträglichere Gefahr, dass der Kronzeuge versuchen wird, sich auf Kosten anderer zu entlasten, so dass Unschuldige dem Risiko eines Fehlurteils ausgesetzt werden. ${ }^{26}$ Die Erfahrungen in Deutschland bestätigen solche

20 Kempf, StV 1999. 67; vgl. dazu näher: Körner, Betäubungsmittelgesetz, 5. A. 2001, § 31 Rn. 30.

21 Vgl. zum Problem der gescheiterten Absprache: Schünemann, Wetterzeichen vom Untergang der deutschen Rechtskultur, 2005, S.26 f.

22 Denny, ZStW 103 (1991), 269 (277); Jeßberger, Kooperation und Strafzumessung, S.89; Mehrens in: Militello u.a. (Hrsg.), Organisierte Kriminalität als transnationales Phänomen, 2000, S.300 f.; Schlüchter, ZRP 1997, 65 (69).

23 S. bzgl. der Vorgängerregelungen bereits kritisch: Hassemer, StV 1989, 80. Die Qualität der Zeugenschutzprogramme wird im wissenschaftlichen Schrifttum unterschiedlich beschrieben. Krit. zur Möglichkeit des Schutzes von Tätern aus dem Bereich der organisierten Kriminalität: Mehrens in: Militello u.a. (Hrsg.), a.a.O., S.305. Positivere Bewertung bei: Kinzig, Die rechtliche Bewältigung von Erscheinungsformen organisierter Kriminalität, 2004, S.769 f.

24 Vgl. BGH, NStZ 1989, 77; OLG Stuttgart, JZ 1992, 537 (538); OLG Hamburg, NStZ 1997, 443; Fezer in: Eser u.a. (Hrsg.), FS für Lenckner, 1998, S.682.

25 Peters, Strafprozessrecht, 4. A. 1985, S.403 f.

26 S. BGH, NStZ 2004, 691 (692); BGH, NStZ-RR 2003, 245; BGH, StV 1995, 62; OLG Koblenz, StV 2007, 71; Burhoff, Handbuch für die strafrechtliche Hauptverhandlung, 5. A. 2007, Rn. 589; Dahs, NJW 1995, 553 (557); Endriß, StraFo 2004, 151 (153); Gössner, Neue Kriminalpolitik 2/ 2000, 5 (6); Kühne, Strafprozessrecht, 7.A. 2007, Rn.800; Mühlhoff / Mehrens, Das Kronzeugengesetz im Urteil der Praxis, 1999, S.98 f.; Paeffgen, StV 1999, 625 (627); Ransiek, StV 1996, 446 (449); Weider, StV 2003, 265. 
Befürchtungen. Der Kronzeuge will einer »Erwartungshaltung der ihm Schonung versprechenden Justizorgane « entsprechen. ${ }^{27} \mathrm{Da}$ er nicht überblickt, welche Informationen die Strafverfolgungsbehörden noch benötigen, er jedoch gerade solche neuen Informationen liefern muss, um in den Genuss der Strafmilderung zu gelangen, besteht die latente Versuchung, tatsächlich begangenen Taten weitere hinzuzufügen oder diese aufzubauschen. ${ }^{28}$ In der Praxis ist zudem zu beobachten, dass Kronzeugen auf Grund ihres äußerlich angepassten Verhaltens gegenüber schweigenden Angeklagten einen Vertrauensvorschuss genießen; anders ausgedrückt, dass ihnen eher geglaubt wird als den durch sie Belasteten. ${ }^{29}$ Dazu ein bekannt gewordenes Beispiel: Der ehemalige Kronzeuge Karl-Peter Pl., der als »Randfigur der RAF-Szene« galt, räumte nach drei Monaten Informationsfluss gegenüber dem »Spiegel« ein, dass er bei den polizeilichen Vernehmungen, bei denen er nicht mit Fakten dienen konnte, Informationen »frei erfunden « habe. U.a. habe er zwei Bekannte als Mitglieder der »Revolutionären Zellen « benannt: »Alles war bis ins Detail erlogen. Ich weiß heute noch nicht, warum ich die beiden als angebliche RZ-Mitglieder belastet habe. Ich war in einem schlimmen Tief.« Als Hintergrund schildert er, dass er sich zwar »Tag für Tag beschissener«, sich aber von den Verhörspezialisten »total abhängig « gefühlt habe. Er habe Angst gehabt, dass sie ihn fallen lassen würden und wollte deshalb »immer irgendwie eine Leistung erbringen. $\ll^{30}$ Dieses exemplarische Beispiel zeigt eine der Hauptproblematiken der Kronzeugenregelung. Da die Kronzeugen nur in ihren Genuss kommen, wenn sie belastende Informationen liefern, nicht aber, wenn sie berichten, dass an einem Verdacht $»$ nichts dran $«$ ist ${ }^{31}$ stehen sie häufig vor der verführerischen Wahl zwischen einer langen Freiheitsstrafe und der falschen Belastung Dritter. Wie hoch der Anteil derjenigen ist, die lügen, lässt sich nur spekulieren. Im wissenschaftlichen Schrifttum besteht jedoch Einigkeit, dass sich die Justiz nicht selten von cleveren Beschuldigten hereinlegen lässt. ${ }^{32}$ Der Einsatz von Kronzeugen bei der Verfolgung von Mitgliedern der IRA in Nord-Irland erwies sich in einem großen Massenverfahren jedenfalls als absoluter »Flop«, da sich die Kronzeugen als derart unglaub-

27 Kühne, Strafprozessrecht, Rn. 800.

28 Nach dem Regierungsentwurf, S.14 soll das Ausmaß der Strafmilderung von der Schwere der offenbarten Taten abhängen.

29 Vgl. aus dem Schrifttum exemplarisch: Mühlhoff / Pfeiffer, ZRP 2000, 121 (122), die Kronzeugenregelung helfe »beim Aufbau eines Vertrauensverhältnisses zwischen dem potentiellen Kronzeugen und den Strafverfolgern, da dann eine klare, Verhandlungsgrundlage' besteht.«

30 Zitiert nach: Gössner, Das Anti-Terror-System, 1991, S.248 f. Ähnliche Schilderungen aus jüngerer Zeit bei: Maurer, Bürgerrechte \& Polizei / Cilip 2001, 21, 24 ff.

31 Krit. Lammer, ZRP 1989, 251 f.; Meyer, ZRP 1976, 25 (27). Dies steht im Widerspruch zu $\S 160$ Abs. 2 StPO, wonach die Staatsanwaltschaft auch entlastende Umstände zu ermitteln hat, s. Hassemer, StV 1986, 550 (552). Teile des wissenschaftlichen Schrifttums sehen auch den Grundsatz der Waffengleichheit im Strafverfahren dadurch verletzt, dass die Belasteten den Kronzeugen nicht gleichsam für entlastende Aussagen belohnen können, s. Mehrens in: Militello u.a. (Hrsg.), Organisierte Kriminalität als transnationales Phänomen, 2000, S.298 m. w. N.

32 Mühlhoff / Pfeiffer, ZRP 2000, 121 (124). 
würdig erwiesen, dass von 65 allein durch sie belasteten Verurteilten, 64 in der Berufungsinstanz freigesprochen werden mussten. ${ }^{33}$

Häufig dürfte es den Gerichten extrem schwer fallen, den Wahrheitsgehalt der Aussagen von Kronzeugen kritisch zu würdigen, da die Kronzeugen als »Insider« zu besonders geschicktem Lügen in der Lage sind. ${ }^{34}$ Am schwierigsten ist es nämlich, Aussagen von Zeugen zu widerlegen, die detailliert überwiegend die Wahrheit sagen und nur Details tatsächlich geschehener Vorgänge wirklichkeitsentfremdet wiedergeben. Die Vorgaben des BGH an die Beweiswürdigung beim Einsatz von Kronzeugen zeigen, dass sich die obergerichtliche Rechtsprechung dieser Problematik bewusst ist. Der Richter muss sich mit der naheliegenden Möglichkeit einer solchen Falschbelas-

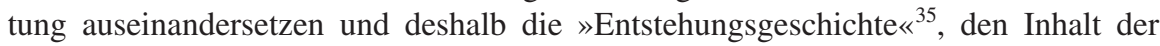
Vereinbarung und sämtliche sonstigen Umstände, die die Entscheidung beeinflussen können, in den Urteilsgründen umfassend darlegen ${ }^{36}$ und würdigen. ${ }^{37}$ Ist ein geständiger Mitbeschuldigter, auf dessen Aussage die Überführung des Angeklagten maßgeblich gestützt werden soll, bereits verurteilt worden, so müssen die Urteilsgründe erkennen lassen, ob sich dieser durch seine Aussage eine Strafmilderung verdient hat oder nicht. ${ }^{38}$ Diese Vorgaben verdienen volle Unterstützung, können die Gefahr von Fehlurteilen jedoch lediglich verringern.

Eine Regelung wie in den USA, wo die freie Beweiswürdigung des Gerichts dadurch eingeschränkt wird, dass eine ausschließlich auf die Aussage eines Kronzeugen gestützte Verurteilung unzulässig ist ${ }^{39}$ ist in dem Entwurf der Bundesregierung nicht vorgesehen. Eine solche Regelung mag dem Grundsatz der freien Beweiswürdigung ( 261 StPO) zwar fremd sein, hätte aber zur Folge, dass sich bekannt gewordene Fälle, in denen Angeklagte ausschließlich auf Grund der Aussagen von Kronzeugen verurteilt wurden, nicht wiederholen. ${ }^{40}$

Die Vorstellung der Bundesregierung, mit einer Erhöhung der Strafdrohungen für falsche Verdächtigungen ( 164 StGB) und dem Vortäuschen einer Straftat $(\S 145 \mathrm{~d}$ StGB) allein ließe sich das Risiko von Falschaussagen verringern, steht im Widerspruch zu den seit Jahrzehnten bekannten Annahmen der empirischen Sanktionsforschung. Diese schreibt der Entdeckungswahrscheinlichkeit, aber vor allem der Normverbindlichkeit, nicht aber bestimmten Strafhöhen Einfluss auf das Legalverhalten der Normadressaten zu. ${ }^{41}$ Dort wo erhebliche Tatanreize geschaffen werden, nützen harte

33 Denny, ZStW 103 (1991), 269, 297 f.; vgl. zu Erfahrungen in Deutschland: Kinzig, a.a.O., S.677 f.

34 Schlüchter, ZRP 1997, 65 (68).

35 BGH, StV 1995, 62.

36 BGH, StV 2003, 264 (265) m. Anm. Weider.

37 OLG Koblenz, StV 2007, 71.

38 BGH, NStZ 2004, $691 \mathrm{f}$.

39 Vgl. dazu: Denny, ZStW 103 (1991), 267, 303 f.

40 Für eine solche Regelung daher: Mühlhoff / Pfeiffer, ZRP 2000, 121 (126); aus Effektivitätsgründen dagegen: Peglau, ZRP 2001, 103 (105).

41 S. exemplarisch: P.A. Albrecht, Kriminologie, 3. A. 2005, 48 ff.; Eisenberg, Kriminologie, 6.A. 2005, S.594 ff.; Kunz, Kriminologie, 4. A. 2004, S.331 ff., 337; Rzepka, KrimJ 2003 , 234 (235). 
Strafen wenig. Ein Kronzeuge, der die Chance sieht, sich durch aufgebauschte oder schlichtweg falsche belastende Aussagen auf Kosten Dritter einer mehrjährigen Haftstrafe zu entziehen, wird sich nicht durch eine solche Strafrahmenanhebung abschrecken lassen. Häufig ist es für das Gericht nämlich kaum nachvollziehbar, was wahr ist und was nicht. ${ }^{42}$ Es besteht hier aber - ebenso wie im Falle einer z.T. geforderten Erweiterung des Katalogs der Wiederaufnahmegründe des § 362 StPO zu Lasten des Täters $^{43}$ - die Gefahr, dass ein reuiger Kronzeuge bei seinen unwahren Aussagen bleibt.

\section{Vereinbarkeit mit dem Amtsermittlungsgrundsatz}

Aus dem Einsatz von Kronzeugen ergeben sich auch Spannungen zum Amtsermittlungsgrundsatz..$^{44}$ Die Staatsanwaltschaft ( $(160 \mathrm{StPO})$ und das Gericht ( $\$ 244$ Abs. 2 StPO) haben die Pflicht, den verfahrensgegenständlichen Sachverhalt umfassend von Amts wegen aufzuklären. Damit die Ermittlungsbehörden die Angaben des Kronzeugen dieser Verpflichtung entsprechend noch überprüfen können, bestimmt $\S 46 \mathrm{~b}$ Abs. 3 des Entwurfs, dass der Kronzeuge sein Wissen vor der Eröffnung des Hauptverfahrens zu offenbaren hat. ${ }^{45}$ Diese Pflicht ergibt sich allerdings - wie gesagt - aus $§ 244$ Abs. 2 StPO auch für das Gericht. Danach hat das Gericht zur Erforschung der Wahrheit die Beweisaufnahme auf alle Tatsachen und Beweismittel zu erstrecken, die für die Entscheidung von Bedeutung sind. Hierbei sind insbesondere die Umstände, die im Rahmen des strafrechtlichen Rechtsfolgenausspruchs zu berücksichtigen sind, im Wege des Strengbeweises zu erforschen. ${ }^{46}$ Da die Fremdbelastungen des Kronzeugen unter den Voraussetzungen des $\S 46 \mathrm{~b}$ E-StGB von erheblicher Bedeutung für das Strafmaß sind, müssen auch diese umfassend auf ihre Richtigkeit überprüft werden. Damit müssten die Gerichte in einer umfassenden Beweisaufnahme inzident sämtliche Aussagen des Kronzeugen prüfen und würdigen, auch wenn diese ganz andere Tatvorwürfe betreffen. Dies würde zu einer massiven Verlängerung der Verfahrensdauer führen. ${ }^{47}$ Dem stehen jedoch die Ziele der Bundesregierung diametral entgegen, durch die Schaffung der Kronzeugenregelung »eine schnelle Aufklärung der Tat« zu ermöglichen und »weitere aufwändige Ermittlungen entbehrlich « zu machen. ${ }^{48}$ Diese Zielsetzung des Gesetzgebers hat sich die Rechtsprechung bei der Anwendung der bisherigen Regelungen bereits dankbar zu eigen gemacht. Das OLG Hamburg führt dazu aus: »Es besteht die Gefahr, dass die nach

42 Endriß, StraFo 2004, 151 (155).

43 Mühlhoff / Pfeiffer, ZRP 2000, 121 (126); Heister-Neumann, DRiZ 2004, 102; Peglau, ZRP 2001, 103 (105); vgl. auch: Kastner in: Lange (Hrsg.), Wörterbuch zur Inneren Sicherheit, 2006, S.185; krit. bereits: Kintzi, DRiZ 2004, 100 (101).

44 S. dazu ausführlich: Fezer in: Eser u.a. (Hrsg.), FS für Lenckner, 1998, S.681 ff.

45 S. Entwurf, S.14 f.

46 Meyer-Goßner, 50. A. 2007, § 244 Rn. 6.

47 S. bereits: Hassemer, StV 1986, 550 (552); ders., StV 1989, 80.

48 So der beschlossene Entwurf der Bundesregierung, S.7; vgl. auch: Mühlhoff / Pfeiffer, ZRP 2000, 121 (122): »Auf Grund des steigenden Arbeitsanfalls der Justiz, ihrer oftmals unzureichenden personellen und sachlichen Ausstattung sowie der Zunahme komplexer Großverfahren (oftmals mit Auslandsbezug) in Sachen der organisierten Kriminalität besteht eine erhöhte Bereitschaft, durch Absprachen mit Kronzeugen schneller und in der Regel auch revisionssicherer zu einer Verfahrenserledigung zu kommen (...).« 
$\S \S 244$ ff. StPO nötige Einvernahme der mit den Ermittlungen zur fremden Tat befassten Kräfte der Strafverfolgungsorgane den mit der Kronzeugenregelung verfolgten Zweck einer verbesserten Aufklärungs- und Ergreifungsmöglichkeit empfindlich stört. Im Fall, dass die weiteren Täter oder Teilnehmer ergriffen sind, würde das Strengbeweisverfahren dem Gericht auch abverlangen, das Offenbarte dadurch zu überprüfen, dass große Teile der Beweisaufnahme eines anderen und regelmäßig sehr umfangreichen Verfahrens durchgeführt oder wiederholt würden. Schon der damit notwendigerweise verbundene Mehraufwand einer gerichtlichen Tatsachenfeststellung in mehreren Verfahren zur gleichen Tat, die nicht einmal zu einer Bindungswirkung über das jeweilige Verfahren hinaus führt, zeigt auf, dass dies vom Gesetzgeber nicht gewollt ist. ${ }^{49}$ Dies ist auch Auffassung des BGH, der hervorhebt, dass der Tatrichter nicht zu einer Überprüfung der Behauptungen des Kronzeugen im Wege einer Beweisaufnahme verpflichtet sei. ${ }^{50}$ So sei ein honorierbarer Aufklärungserfolg im Sinne von $\S 31$ BtMG bereits eingetreten, wenn auf Grund der Angaben des Aufklärungsgehilfens zur Überzeugung des Gerichts bestimmte identifizierbare Personen hinreichend verdächtig seien. ${ }^{51}$ Nach der ständigen Rechtsprechung des BGH soll es deshalb ausreichen, dass seine Schilderung der Beteiligung Dritter zu einem »voraussichtlich erfolgreichen Abschluss der Strafverfolgung beiträgt, $«^{52}$ wobei es nach Auffassung des OLG Stuttgart bereits genüge, wenn die Aufklärungsmöglichkeit verbessert werde. ${ }^{53}$ Daher sei das Gericht auch nicht verpflichtet, abzuwarten, bis die Strafverfolgungsbehörden entsprechende Ermittlungen durchgeführt haben. ${ }^{54}$ Wie die Gerichte aber auf hinreichend nachvollziehbarer Grundlage zu der eigenen Überzeugung gelangen wollen, dass die Angaben des Kronzeugen der Wahrheit entsprechen, bleibt offen. Auch durch die Neuregelung besteht deshalb die Gefahr, dass sich die Staatsanwaltschaft und die Gerichte zu sehr auf die Aussagen des Kronzeugen verlassen und andere erzielbare Beweismittel vernachlässigen. ${ }^{55}$

\section{Vereinbarkeit mit dem Legalitätsprinzip}

Die Kronzeugenregelung kann im Einzelfall zur Folge haben, dass Täter, die einer schweren Tat überführt werden können, gleichwohl nicht verurteilt werden. Eine solche Vorgehensweise kollidiert mit dem im deutschen Strafverfahren geltenden Legalitätsprinzip. Dieses in $\S 152$ Abs. 2 StPO niedergelegte Prinzip bestimmt die Pflicht der Staatsanwaltschaft, ein Strafverfahren einzuleiten, wenn zureichende tatsächliche Anhaltspunkte für einen Gesetzesverstoß vorliegen. ${ }^{56}$ Das Legalitätsprinzip hat in

49 OLG Hamburg, NStZ 1997, 443 (444); so auch: Meyer-Goßner, 44. A. 1999, Art. 4 § 1 KronzG Rn.5.

50 BGH, NStZ 1998, 90.

51 BGHSt 31, 163 (166); BGH, StV 1994, 543; BGH, NStZ 1989, 580 m. Anm. Weider; s. auch: Körner, Betäubungsmittelgesetz, § 31 Rn. 31.

52 BGH, StV 1985, 14; BGH, NStZ 1989, 77; vgl. auch: OLG Hamburg, NStZ 1997, 443.

53 OLG Stuttgart, JZ 1992, 537 (538).

54 BGH, StV 1994, 544.

55 S. schon zum KronzG: Bocker, Der Kronzeuge, S.91; Denny, ZStW 103 (1991), 269 (274); Fezer in: Eser (Hrsg.), FS für Lenckner, S.681 (697); Lammer, ZRP 1989, 248 (251).

56 Meyer-Goßner, StPO, 50. A. 2007, § 152 Rn. 2; Naucke, Strafrecht, 10. A. 2002, S.175. 
Deutschland eine lange Tradition. Bereits Kant nannte das Strafgesetz einen »kategorischen Imperativ « und brachte damit zum Ausdruck, dass in einer gerechten Strafrechtspflege das Strafgesetz unabhängig von bloßen Nützlichkeitserwägungen unbedingt angewandt werden müsse. ${ }^{57}$ Das Legalitätsprinzip gehört zu den fundamentalen Prinzipien der Strafprozessordnung. Es soll eine gleichmäßige und willkürfreie Rechtsanwendung gewährleisten ${ }^{58}$ und genießt über Art. 3 Abs. 1 GG und das Rechtsstaats- und Gesetzlichkeitsprinzip Verfassungsrang. ${ }^{59}$ Auch wenn das Legalitätsprinzip inzwischen durch zahlreiche Opportunitätsregelungen ( $\S 153$ ff. StPO) relativiert worden ist, besteht doch weitgehende Einigkeit, dass es jedenfalls im Bereich der schweren Kriminalität uneingeschränkt zur Anwendung gelangen muss. ${ }^{60}$ Vergegenwärtigt man sich mit welcher Akribie auch Straftaten im Bagatellbereich zur Anklage gebracht und mitunter empfindlich geahndet werden, ${ }^{61}$ ist ein von der Bundesregierung ausdrücklich genannter vollständiger Strafverzicht im Fall eines schweren Raubes $(\S \S 249,250 \mathrm{StGB})^{62}$ mit dem dem Legalitätsprinzip zugrunde liegenden Gebot der gleichmäßigen Strafverfolgung nicht zu vereinbaren. Vor allem wenn man sich vorstellt, dass ein Kronzeuge auf freien Fuß gesetzt wird, während seine Mittäter, die u.U. wesentlich geringere Tatbeiträge geleistet haben, mehrjährige Freiheitsstrafen verbüßen müssen, wird deutlich, dass eine solch weitgehende Aufweichung des Legalitätsprinzips zugunsten von Opportunitätserwägungen nicht mehr akzeptabel ist. ${ }^{63}$

\section{Vereinbarkeit mit dem Öffentlichkeits- und Fair Trialgrundsatz.}

Weitere Bedenken bestehen dagegen, dass sich der Kronzeuge gem. §46b Abs. 3 EStGB bereits vor der Eröffnung des Hauptverfahrens zur Kooperation entscheiden muss, so dass die Kooperationsvereinbarung außerhalb der Hauptverhandlung stattfindet. Damit wird der Eindruck erweckt, als ob die Strafrechtspflege die Öffentlichkeit scheue. Dies ist gerade bei schweren Tatvorwürfen ausgesprochen heikel. Die Rechtsprechung hat beim »Deal« darauf reagiert und fordert, dass der wesentliche Inhalt der Absprachen in die Hauptverhandlung eingeführt werden müsse. ${ }^{64}$ Der Vorwurf der

57 Kant, Metaphysik der Sitten, Akademie Ausgabe Band VI, 1902, S.331.

58 BVerfG, NStZ 1982, 430; P. A. Albrecht, Kriminologie, 3. A. 2005, S.115 ff.; Hassemer, StV 1986, 550 (552); Weigend, ZStW 113 (2001), 271 (284).

59 Hoyer, JZ 1992, 233 (235); Naucke, Strafrecht, S.175; sowie umfassend: Pott, Die Außerkraftsetzung der Legalität durch das Opportunitätsdenken, 1996, S.10 ff.

60 S. Gössel, Gutachten C zum 60. DJT 1994, S. C 31. Zur Unvergleichbarkeit der $§ \S 153$ ff. StPO und der Kronzeugenregelung: Hassemer, StV 1986, 550 (552). Unzutreffend: Hilger, NJW 1989, 2377 (2378).

61 S. dazu Köhler, JZ 1989, 697; Thoss, KritV 1994, 392 ff.

62 S. Pressemitteilung des Bundesjustizministeriums v. 16.5.2007.

63 So schon zu den Vorgängerregelungen: Beulke, Strafprozessrecht, Rn. 342; Dahs, NJW 1995, 553 (557); Meyer-Goßner, 44. A. 1999, Vor KronzG Rn. 7; Neumann, StV 1994, 273 (276); KK-StPO / Pfeiffer, 4. A. 1999, Einleitung Rn. 32b; Pott, a.a.O., S.115; Roxin, Strafverfahrensrecht, 25. A. 1998, S.90; Schaefer, NJW 2000, 2325 (2326); Volk, NJW 1996, 879 (881); Weigend, ZStW 109 (1997), 103 (114); vgl. auch Erdem, Forum Recht 2007, 64 (65); Kühl, NJW 1987, 737 (744).

64 BGHSt 43, 195 ff. 
»Mauschelei« und fehlenden Transparenz ist beim Einsatz von Kronzeugen auch nach der geplanten Neuregelung noch gravierender. Es handelt sich hierbei nämlich um einen »Vergleich (auch) zu Lasten Dritter $\ll .{ }^{65}$ Der BGH setzt deshalb auch beim Einsatz von Kronzeugen zu Recht voraus, dass der Hintergrund und der Inhalt der Vereinbarung - insbesondere im Verfahren gegen belastete Dritte - detailliert zu schildern sind. ${ }^{66}$ Die Tendenz, die Hauptverhandlung zugunsten des Ermittlungsverfahrens zu entwerten, setzt sich jedoch fort. ${ }^{67}$ Wenn das Verfahren gegen den Kronzeugen eingestellt wird, findet gegen diesen überhaupt keine Hauptverhandlung statt. ${ }^{68}$ Solche Gespräche außerhalb der Hauptverhandlung hinsichtlich der Einstellung des - abgetrennten - Verfahrens erfolgen ohne die Anwesenheit der Verteidiger der Belasteten, wodurch regelmäßig bereits Weichen für das Verfahren gegen die Belasteten gestellt werden. ${ }^{69}$

Der Kronzeuge ist in der Hauptverhandlung gegen die Belasteten zu vernehmen. Laut Meyer-Goßner könne aber das Bedürfnis, den Kronzeugen vor den Beschuldigten zu schützen, dazu führen, dass er entsprechend $§ 96$ StPO geheimgehalten werde, falls Maßnahmen nach $\S 68$ S.1 StPO, § 247 StPO oder $§ 172$ Nr. 1 GVG nicht ausreichten. In diesem Fall sei bei einer Einführung seines Wissens durch einen Dritten für eine Verurteilung erforderlich, dass die Angaben durch andere Beweismittel gestützt werden. ${ }^{70}$ Solche zusätzlichen Indizien, die die Behauptungen des Kronzeugen stützen, dürften allerdings häufig zu finden sein, ohne dass aus ihnen allein ein zwingender Schluss gezogen werden kann.

Weder der Rechtsprechung noch der Kommentarliteratur ist die Beurteilung der Konstellation zu entnehmen, dass der Kronzeuge von der Verteidigung weder im Rahmen einer richterlichen Vernehmung außerhalb noch in der Hauptverhandlung vernommen werden konnte. In diesem Fall ist eine effektive Verteidigung gegen die Beschuldigungen des Kronzeugen jedoch nicht möglich, so dass eine Verwertung der Aussagen des Kronzeugen zu Lasten des Beschuldigten den durch Art. 6 EMRK garantierten Anspruch auf ein faires Verfahren verletzen würde.

Diese Einschränkung des Öffentlichkeitsgrundsatzes und die Gefährdungen einer effektiven Strafverteidigung vermögen für sich allein betrachtet nicht ausreichen, die Verfassungswidrigkeit der Neuregelung zu begründen, stärken jedoch die Vorbehalte gegen die Kronzeugenregelung.

65 Mühlhoff / Pfeiffer, ZRP 2000, 121 (125); Endriß, StraFo 2004, 151 (154); vgl. dazu auch: Sinner, Der Vertragsgedanke im Strafprozessrecht, 1999, S.227 ff.

66 BGH, StV 2003, 264 (265) m. Anm. Weider.

67 Vgl. Meyer-Goßner, 44. A. 1999, Vor KronzG Rn. 7; Mühlhoff/ Pfeiffer, ZRP 2000, 121 (126); Hassemer, StV 1989, 80; KK-StPO / Pfeiffer, Einl. Rn. 32b.; Schlüchter. ZRP 1997, 65 (68); s. hierzu auch: Schünemann, Wetterzeichen vom Untergang der deutschen Rechtskultur, S.31; Weigend, ZStW 113 (2001), 271 (275).

68 Vgl. Peglau, ZRP 2001, 103 (104).

69 Vgl. Hassemer, StV 1986, 550 (552). Anders aber im Regelfall gem. § 268c Abs. 2,5 StPO im Verfahren gegen den Beschuldigten, s. BGH, NStZ 1999, 417.

70 Meyer-Goßner, 44. A. 1999, Art. 4 § 1 KronzG Rn. 15; vgl. auch: Hilger, NJW 1989, 2377 (2378); Löwe-Rosenberg / Gollwitzer, 25. A. 1999, § 251 Rn. 40; krit. Böcker, Der Kronzeuge, S.93; Endriß, StraFo 2004, 151 (154); Hassemer, StV 1986, 550 (552); Kunert / Bernsmann, NStZ 1989, 449 (458); Schlüchter, ZRP 1997, 65 (68) 


\section{Rechtfertigung des Strafnachlasses}

Fragwürdig ist überhaupt, wie eine solch weitgehende Vergünstigung gerechtfertigt werden könnte. Es stellt sich die grundsätzliche Vereinbarkeit der Neuregelung mit dem Schuldprinzip. ${ }^{71}$ Der Entwurf wirft diese Frage nur kurz auf, lässt sie jedoch dahinstehen: »Die entsprechende Rechtsfertigung kann sich jedenfalls aus der ebenfalls aus dem Rechtsstaatsprinzip abzuleitenden wesentlichen Aufgabe des Staates ergeben, gerade schwere Straftaten aufzuklären und zu verhindern. ${ }^{72}$ Eine solche Begründung verkennt jedoch den im Rechtsstaat geltenden fundamentalen Unterschied von Aufgaben und Befugnissen.

Gegen ein Rechtfertigungserfordernis kann zudem nicht eingewandt werden, bei der Kronzeugenregelung gehe es lediglich um eine den Kronzeugen ausschließlich begünstigende und damit nicht rechtfertigungsbedürftige Schuldunterschreitung, da eine solche Unterschreitung über Art. 3 Abs. 1 GG Grundrechtsrelevanz für Dritte erlangt. ${ }^{73}$

\section{a) Rücktritt vom Versuch}

Zum Teil werden zur Rechtfertigung des Strafnachlasses Parallelen zur Rücktrittsregelung des $\S 24$ StGB herangezogen, da der Kronzeuge wie beim Rücktritt »Partei ergreife für die Kräfte, die der deliktischen Unternehmung entgegenstehen. ${ }^{74}$ Eine solche Argumentation kann jedoch gleich aus mehreren Gründen nicht überzeugen. Der Grund der Strafaufhebung durch den Rücktritt vom Versuch gilt bis heute als nicht hinreichend geklärt. ${ }^{75}$ Von den Befürwortern der Kronzeugenregelung behauptete Parallelen ließen sich allenfalls ziehen, wenn man das Rücktrittsrecht ausschließlich »kriminalpolitisch « unter Rückgriff auf die Vorstellung begründen würde, dem Täter eine »goldene Brücke ${ }^{76}$ zum Recht bauen zu wollen. ${ }^{77}$ Hierbei müsste jedoch bereits darüber hinweggesehen werden, dass der Rücktritt nur im Versuchsstadium möglich ist.

71 Da Kronzeugen regelmäßig erst zu einem Zeitpunkt Aussagen machen, in denen sie bereits längere Zeit mit den Tatvorwürfen konfrontiert werden - laut Gössner, Neue Kriminalpolitik 2/ 2000, 5 (6) in der Untersuchungshaft - ist nicht zu erwarten, dass durch ihre Aussagen noch konkret geplante Straftaten verhindert werden können, so im Ergebnis auch: Denny, ZStW 103 (1991), 269 (279); Hassemer, StV 1986, 550 (552); Ders., StV 1989, 80. Maurer, Bürgerrechte \& Polizei / Cilip 2001, 20 (27), konstatiert, dass das erklärte Ziel der Straftatenverhinderung verfehlt wurde. Die Legitimität des Strafnachlasses oder Absehens von Strafe konzentriert sich daher in erster Linie auf die Strafverfolgungsebene.

72 Entwurf, S.9.

73 Zur Frage, ob die Schuld aus präventiven Erwägungen unterschritten werden darf: Dafür: Roxin, Strafrecht AT I, 4. A. 2006, S.91 ff. Schönke / Schröder - Stree, Vor §§ 38 Rn. 18a. Dagegen: BGHSt 24, 132 (134); Jescheck / Weigend, Strafrecht AT, 1996, S.879 f.

74 Behrendt, GA 1991, 337 (338); vgl. auch: Buttel, Kritik der Figur des Aufklärungsgehilfens im Betäubungsmittelstrafrecht, 1988, S.228, 252 ff.; Hoyer, JZ 1994, 233 (240).

75 Köhler, Strafrecht AT, 1997, S.470; Kühl, Strafrecht AT, § 16 Rn. 4; Tröndle / Fischer, 54. A. 2007, § 24 Rn. 2; s. zur neusten Diskussion: NK-StGB / Zaczyk, 2. A. 2005, § 24 Rn. 1 ff.; Yamanaka in: Schünemann u.a. (Hrsg.), FS für Roxin, 2001, S.773 ff.

76 Franz von Liszt, Lehrbuch des deutschen Strafrechts, 14. u. 15. A. 1905, S.211.

77 Krit. zur »kriminalpolitischen Theorie« des Rücktritts: Lang-Hinrichsen in: Bockelmann u.a. (Hrsg.), FS für Engisch, 1969, S.368; Schönke / Schröder-Eser, 27. A. 2006, § 24 Rn. 2; Wessels / Beulke, Strafrecht AT, 36. A. 2006, S.227 f., die die Wirklichkeitsferne dieser Theorie bemängeln. 
Und dies ist nicht ohne Grund der Fall: Überzeugender als die einseitig strafzweckorientierten Theorien sind nämlich diejenigen Ansätze, die Versuch und Rücktritt als einheitlichen Vorgang begreifen und die Strafaufhebung beim Rücktritt ausgehend vom Strafgrund des Versuchs rechtfertigen wollen. ${ }^{78}$ Es ist dem Täter im Versuchsstadium nämlich noch möglich, dass vom ihm verwirklichte Unrecht und seine Schuld dadurch weitgehend zu annullieren, dass er durch einen freiwilligen Umkehrprozess die weitere Ausführung der Tat aufgibt oder die Vollendung der Tat verhindert. ${ }^{79}$ Von alldem kann jedoch beim Kronzeugen nicht die Rede sein. Es sind vor allem keine »autonomen Motive«, die ihn zur Kooperation mit der Strafjustiz zu einem Zeitpunkt motivieren, wenn er mit Tatvorwürfen konfrontiert wird. ${ }^{80}$ Zudem ist ein Rücktritt als persönlicher Strafaufhebungsgrund nur von eigenen Taten möglich. Das zentrale Motiv der Kronzeugenregelung besteht jedoch darin, Beweise hinsichtlich der Taten Dritter zu erlangen.

\section{b) Tätige Reue}

Andere Ansätze versuchen, die Kronzeugenregelung unter Rückgriff auf die Rechtsfigur der tätigen Reue zu rechtfertigen. ${ }^{81}$ Doch auch ein solches Vorgehen kann nicht überzeugen. Dieser persönliche Strafmilderungs- und -aufhebungsgrund spielt im heutigen Strafrecht vorwiegend dort eine Rolle, wo der Gesetzgeber einen frühen Vollendungszeitpunkt gewählt hat. ${ }^{82}$ Der Täter kommt beispielsweise bei den Brandstiftungsdelikten gem. $\S 306 e$ StGB zudem nur dann in den Genuss des Strafverzichts oder Strafnachlasses, wenn er freiwillig den Brand löscht bevor erheblicher Schaden entsteht. Der Gesetzgeber hat sich bewusst dagegen entschieden, eine allgemeine Regelung der tätigen Reue zu schaffen. Es ist zweifelhaft, ob sich der Rechtsgedanke auf sämtliche Straftatbestände übertragen lässt. Dort wo keine Regelungen der tätigen Reue bestehen, wird das Bemühen um Schadenswiedergutmachung im Rahmen der Strafzumessung als Nachtatverhalten ( $\S 46$, 46a StGB) honoriert. Von alldem kann jedoch im Falle des Kronzeugen nicht der Rede sein, da der Strafnachlass hier nicht davon abhängt, dass er einen von ihm angerichteten materiellen oder immateriellen Schaden zumindest teilweise wiedergutmacht oder sich bei den Opfern entschuldigt. Zudem wird der Kronzeuge regelmäßig erst mit der Strafrechtspflege kooperieren, wenn diese ihn mit der eigenen Tatverstrickung konfrontiert. ${ }^{83}$

78 Köhler, Strafrecht AT, S.468 f.; Lang-Hinrichsen in: Bockelmann (Hrsg.), FS für Engisch, S.353, 369 ff.; Murmann, Versuchsunrecht und Rücktritt, 1999, S.27 ff.; Welzel, Das deutsche Strafrecht, 9. A. 1965, S.176; NK-StGB/Zaczyk, 2. A. 2005, § 24 Rn. 5 und wohl auch Schönke / Schröder-Eser, § 24 Rn. 3.

79 NK/ Zaczyk, § 24 Rn. 5: »So wie die Tatschuld Entschluss zur Unrechtsmaxime ist, ist die (nur beim Versuch mit dieser Wirkung mögliche) Rückkehr zur Rechtsmaxime Aufhebung des ersten Entschlusses (...).«

80 Vgl. Schönke / Schröder-Eser, § 24 Rn. 67.

81 Vgl. Buttel, a.a.O., S.227 ff.; Körner, Betäubungsmittelgesetz, § 31 Rn. 2.

82 Jescheck / Weigend, Strafrecht AT, S.899.

83 Vgl. auch: Kempf, StV 1999, 67; Lenckner / Sternberg-Lieben in: Schönke / Schröder, 27. A. 2006, § 129a Rn. 1a; Mushoff, Forum Recht 2006, 80; KK-StPO / Pfeiffer, Einl. Rn. 32b; Weigend in: Vogler (Hrsg.), FS für Jescheck, 1985, S.1346. Das eigennützige Motiv des Kronzeugen zur Kooperation hat im Rahmen der Versagung der tätigen Reue außer acht zu bleiben, da das Recht nicht die Aufgabe hat, Gesinnungen zu überprüfen (s. dazu jüngst: Hirsch, JZ 2007, 494 (499)), sondern bei der tätigen Reue lediglich rechtskonformes (äußeres) Verhalten honoriert, vgl. im Ergebnis auch: Koumbarakis, a.a.O., S.69. S. zum gleichen Problem beim Rücktritt: BGH, StV 2007, 72; Köhler, Strafrecht AT, S.481 f. 


\section{c) Kooperationsbereitschaft als Strafmilderungsgrund}

Ein wesentliches Motiv für den Strafnachlass bei der Kronzeugenregelung liegt wohl eher darin, den Täter für seine Kooperationsbereitschaft zu belohnen.$^{84}$ Gem. $§ 46$ Abs. 2 S.2 StGB hat das Gericht auch das Nachtatverhalten des Täters zu berücksichtigen, was dafür sprechen könnte, dass eine Zusammenarbeit mit der Strafjustiz strafmildernd zu honorieren ist. Allerdings wird von der Rechtsprechung nur solches Nachtatverhalten strafmildernd einbezogen, das Rückschlüsse auf eine geringere »rechtsfeindliche Gesinnung « des Täters zum Zeitpunkt der Tat zulasse. ${ }^{85}$ Anerkannt ist beispielsweise die Möglichkeit, ein Geständnis unter dieser Voraussetzung strafmildernd zu berücksichtigen. ${ }^{86} \mathrm{Ob}$ Geständnisse im Strafverfahren Einfluss auf die Tatschuld eines Monate oder gar Jahre zurückliegenden Verbrechens haben, wird in jüngerer Zeit bezweifelt. ${ }^{87}$ Auch sind die Spannungen der strafmildernden Berücksichtigung des Geständnisses zur Freiheit von Selbstbelastungszwang des Angeklagten nicht zu übersehen. ${ }^{88}$ Diese grundsätzlichen Fragen ${ }^{89}$ können jedoch bezüglich der Vereinbarkeit der Kronzeugenregelung mit dem Schuldprinzip dahinstehen, da bei ihr nicht in erster Linie die Eigen-, sondern die Fremdbelastung honoriert werden soll. Weshalb die Belastung eines Dritten, die eigene Tatschuld annullieren soll, ist nicht ersichtlich. ${ }^{90}$ Auch hebt der BGH hervor, dass ein Geständnis nicht zu einem Strafmaß führen darf, das dem Unrechtsgehalt der Tat nicht mehr gerecht wird. ${ }^{91}$ Dieser Grundsatz gilt auch für die Kronzeugenregelung. Es ist aber höchst zweifelhaft, ob die gesetzliche Milderungsmöglichkeit in § 46b Abs. 1 E-StGB dem Gebot schuldangemessenen Strafens noch entspricht. Eine Strafrahmenverschiebung nach § 49 Abs. 1 StGB

84 Vgl. krit. Jescheck / Weigend, Strafrecht AT, S.898.

85 BGHSt 1, 105 f.; weitere Nachweise bei: Bruns, Das Recht der Strafzumessung, 1985, S.231. Diese »Indizienkonstruktion « wurde in jüngerer Zeit mit Recht als spekulativ angegriffen, da es dem Gericht nicht möglich ist, im Rahmen der Hauptverhandlung die Motive des Täters zum Zeitpunkt der Tat zu überprüfen, s. Frisch in: Roxin (Hrsg.), BGH-Festgabe, 2000, S.292 ff.; Jescheck / Weigend, Strafrecht AT, S.894; s. hierzu auch: Bruns, Neues Strafzumessungsrecht, 1988, S.60.

86 BGHSt 43, 195 (209); weitere Nachweise bei: Tröndle / Fischer, 54. A. 2007, § 46 Rn. $50 \mathrm{ff}$.

87 Jescheck / Weigend, Strafrecht AT, S.893 f.

88 S. aus der neueren Diskussion: Hammerstein, StV 2007, 48 ff.; Hörnle, Tatproportionale Strafzumessung, 1999, S.181 f.; Jescheck / Weigend, Strafrecht AT, S.893 f.; Möller, JR 2005, 314 ff.; Rieß, FS für Christian Richter II, 2006, S.433 ff.; Rode, StraFo 2007, 98 ff.

89 Weiterführend: Frisch in: Roxin (Hrsg.), BGH-Festgabe, S.292 ff.; ders. (Hrsg.), Tatproportionalität, 2003.

90 Da das Gericht von Amts wegen zur Aufklärung des Sachverhalts verpflichtet ist, kommt eine Strafmilderung jedenfalls nicht allein deshalb in Betracht, weil dem Gericht die Arbeit erleichtert wurde, Bruns, Das Recht der Strafzumessung, 2.A. 1985, S.233. Anders jedoch häufig die Praxis, s. Rieß, FS für Chr. Richter II, S.443.

91 BGHSt 43, 195, 208 f. In seiner ersten Anwendung des Kronzeugengesetzes hatte der BGH noch vorsichtiger formuliert, dass der Umfang der vorgesehenen Vergünstigungen »nicht ausschließlich am kriminalpolitischen Zweck dieser Vorschriften ausgerichtet werden « dürfe und - wie im Entwurf in $§ 46 \mathrm{~b}$ Abs. 2 StGB vorgesehen - gefordert, dass der Aufklärungsbeitrag mit der Schwere der Schuld abzuwägen sei, BGH, JZ 1992, 536 (537). 
oder gar ein Absehen von einer eigentlich schuldangemessenen Freiheitsstrafe von drei Jahren lässt sich durch kooperatives Verhalten allein nicht rechtfertigen. ${ }^{92}$

\section{d) Ermittlungsnotstand}

Hoyer will die Kronzeugenregelung über die Rechtsfigur des Ermittlungsnotstands rechtfertigen. Aus Sicht der Strafverfolgungsorgane ähnele die Situation beim Einsatz von Kronzeugen der beim rechtfertigenden Notstand, wo ebenfalls Interessenverletzungen sich dadurch legitimierten, dass zugleich bestimmte (überwiegende) Gegeninteressen gewahrt würden. Bei der Kronzeugenregelung werde wegen eines überwiegenden Interesses an der effektiven Strafverfolgung und an der Verhinderung weiterer Straftaten das Legalitätsprinzip durchbrochen. ${ }^{93} \mathrm{Ob}$ eine solche Konstruktion jedoch auf die weitreichenden Neuregelungen angewendet werden kann, ist aus mehreren Gründen mehr als zweifelhaft. So ist der Figur eines Ermittlungsnotstandes der Grundsatz der Subsidiarität immanent. Das hieße für die Kronzeugenregelung, dass diese nur ausnahmsweise dann zur Anwendung kommen dürfte, wenn alle anderen Instrumente nicht zum Erfolg geführt haben. Die neue Kronzeugenregelung in $\S 46 \mathrm{~b}$ StGB verliert hierzu jedoch kein Wort. Auch aus dem Katalog des $\S 100$ a Abs. 2 E-StPO lassen sich keine entsprechenden Schlüsse ziehen.

Weiterhin sieht sich die Figur des Ermittlungsnotstandes dem Einwand ausgesetzt, dass diese auf reinen Nützlichkeitserwägungen beruht. Verfahrensgerechtigkeit und materielle Gerechtigkeit lassen sich im Rechtsstaat jedoch nicht dem reinen KostenNutzen-Kalkül unterordnen. ${ }^{94}$

Zudem ist der Beweis für die These eines Ermittlungsnotstandes nicht erbracht. ${ }^{95}$ Die Kronzeugenregelung nach altem Recht hatte wenig praktische Bedeutung. ${ }^{96}$ Dies dürfte jedoch weniger mit ihrem im Verhältnis zur heutigen Rechtslage eingeschränkten Anwendungsbereich, sondern mit den strukturellen Problemen der Kronzeugenregelung zusammenhängen. Die bisherigen Erfahrungen im Umgang mit Kronzeugen sind nämlich insgesamt ernüchternd. Sie zeigen, dass es zweifelhaft ist, ob eine allgemeine Kronzeugenregelung zu einer Verbesserung der Ermittlungstätigkeit beitragen kann. ${ }^{97}$ Die Bundesregierung hat zur Begründung der Wiedereinführung der Kronzeu-

92 Gleicher Auffassung hinsichtlich der Vorgängerregelungen: Hassemer, StV 1986, 550 (552); Jeßberger, Kooperation und Strafzumessung, S.65; Weigend in: Vogler (Hrsg.), FS für Jescheck, S.1348.

93 Hoyer, JZ 1994, 233 (240); vgl. auch: Koumbarakis, a.a.O., S.70; Jeßberger, Kooperation und Strafzumessung, S.110; Mehrens in: Militello u.a. (Hrsg.), Organisierte Kriminalität als transnationales Phänomen, S.294; Peglau, ZRP 2001, 103 (104); Schlüchter, ZRP 1997, 65, 69.

94 Harzer, Die Revisionsbegründung »zu Protokoll der Geschäftsstelle«, 1995, S.20 ff.; Hassemer, StV 1986, 550 (553); Lisken, Rechtsstaat - was sonst?, 1996, S.69; Weigend, ZStW 109 (1997), 103 (115).

95 So bereits: Jung, Straffreiheit für den Kronzeugen?, S.102; Kunert / Bernsmann, NStZ 1989, 449 (457). S. aus jüngerer Zeit: Stellungnahme des Strafrechtsausschusses des DAV, StV 2001, 317; Endriß, StraFo 2004, 151 (155); Mehrens in: Militello u.a. (Hrsg.), Organisierte Kriminalität als transnationales Phänomen, S.297.

96 Tröndle / Fischer, 54. A. 2007, § 129a Rn. 33.

97 Dahs, NJW 1995, 553 (557); Kühne, Strafprozessrecht, Rn. 800. 
genregelung vor allem Schwierigkeiten bei der Beweiswürdigung gegenüber Tätern aus den Bereichen des Terrorismus und der organisierten Kriminalität angeführt. ${ }^{98}$

In der Zeit von 1989 bis 1999 wurde die Kronzeugenregelung in Deutschland in Verfahren gegen Terroristen etwa 20 bis 25 mal angewendet. ${ }^{99}$ Die Ermittlungserfolge gegen Mitglieder der RAF nach der Wiedervereinigung sind in keinem Fall auf die Angaben von Kronzeugen zurückzuführen, sondern beruhen auf der Ausweitung der Zugriffsmöglichkeiten auf die in der ehemaligen DDR lebenden Aussteiger. Soweit sich die Ergriffenen bereit erklärten, mit den Strafverfolgungsbehörden zu kooperieren, dürfte diese Bereitschaft weniger auf die versprochenen Strafmilderungen, sondern vor allem darauf zurückzuführen sein, dass die Aussteiger mit den Zielen des linken Terrorismus vollständig gebrochen hatten. ${ }^{100}$ Verhaftete aktive Mitglieder der RAF haben eine Zusammenarbeit mit den Strafverfolgungsbehörden stets abgelehnt, da sie ein solches Verhalten als »Verrat« auffassten. Politische oder religiöse Überzeugungstäter sind keine geeigneten Adressaten der Kronzeugenregelung. ${ }^{101}$

Zwingende Argumente für den Einsatz der Kronzeugenregelungen liefern auch nicht die Erfahrungen, die mit ihr im Umgang mit Mitgliedern der »organisierten Kriminalität« erzielt wurden. Bislang sind Kronzeugen in diesem Bereich in der Zeit von 1994 bis 1999 schätzungsweise in maximal 25 Fällen ${ }^{102}$ aufgetreten und waren nicht in der Lage, Mafia-Strukturen zu zerschlagen. ${ }^{103}$ Zieht man die Erfahrungen heran, die mit der bereits im Jahr 1981 eingeführten »kleinen Kronzeugenregelung« nach $§ 31$ BtMG gemacht wurden, so fällt die Bilanz nur wenig vielversprechender aus. ${ }^{104} \mathrm{Ob}$ wohl von dieser Regelung seit ihrer Einführung ein weitreichender, geradezu »inflationärer « Gebrauch gemacht wurde ${ }^{105}$, ist es auch mit ihr bis heute nicht gelungen, organisierte Drogenringe zu zerschlagen. Nicht die »großen Dealer« konnten überführt werden, sondern vorwiegend kleine und mittlere Drogendelikte wurden durch sie aufgeklärt. ${ }^{106}$ Nicht mehr nachträglich nachvollziehen lässt sich jedoch in diesen Fällen, ob es nicht auch auf andere Weise gelungen wäre, die betreffenden Täter zu überfüh-

98 Vgl. den verabschiedeten Entwurf der Bundesregierung, Gesetz zur Änderung des Strafgesetzbuches - Strafzumessung bei Aufklärungs- und Präventionshilfe, S.1.

99 Mühlhoff / Pfeiffer, ZRP 2000, 121 (123).

100 Hoyer, JZ 1994, 233 (234); Maurer, Bürgerrechte \& Polizei / Cilip 2001, 20 (25); KK-StPO / Pfeiffer, Einl. Rn. 32b.

101 Denny, ZStW 103 (1991), 269 (276); Hassemer, StV 1986, 550 (551); Hoyer, JZ 1994, 233 (234); Scheerer, StV 1989, 72 (83).

102 Mühlhoff / Mehrens, Das Kronzeugengesetz im Urteil der Praxis, 1999, S.97.

103 Gössner, Neue Kriminalpolitik 2 / 2000, 5 (6).

104 Krit. Eisenberg, Kriminologie, 6. A. 2005, § 45 Rn. 133; Endriß, StraFo 2004, 151 ff.; Hassemer, StV 1986, 550 (551); Jescheck / Weigend, Strafrecht AT, S.899.

105 Schlüchter, ZRP 1997, 65 (71); vgl. auch: Körner, Betäubungsmittelgesetz, § 31 Rn. 2. Mühlhoff / Mehrens, Das Kronzeugengesetz im Urteil der Praxis, 1999, S.97, berichten von über 6.000 Anwendungsfällen.

106 Fezer in: Eser u.a. (Hrsg.), FS für Lenckner, S.694; Körner, Betäubungsmittelgesetz, § 31 Rn. 4; vgl. auch: Eisenberg, Kriminologie, § 45 Rn. 133. 
ren. ${ }^{107}$ Hier mag der Einsatz von Kronzeugen mitunter auch nützlich gewesen sein. Ein die Kronzeugenregelung legitimierender Ermittlungsnotstand lässt sich durch solche Nachweise nicht begründen. Demgegenüber überwiegen die Berichte, nach denen der Einsatz von Kronzeugen dazu geführt hat, dass Unschuldige mit den Belastungen eines Strafverfahrens überzogen wurden.

\section{e) Zusammenfassung}

Es sind somit ausschließlich vordergründige Nützlichkeitserwägungen, die zur Rechtfertigung der Kronzeugenregelung angeführt werden können. ${ }^{108}$ Die »Funktionstüchtigkeit der Strafrechtspflege $\ll{ }^{109}$ zeichnet sich jedoch nicht ausschließlich dadurch aus, dass Strafverfahren rechtskräftig mit einer Verurteilung abgeschlossen werden, sondern setzt vielmehr bei dem Ziel, die Wahrheit aufzuklären und eine gerechte Sachentscheidung zu fällen, die Einhaltung rechtsstaatlicher Verfahrensprinzipien voraus. Nur eine faire und gerechte Strafrechtspflege ist eine funktionstüchtige Strafrechtspflege.

Grundlage der Strafzumessung ist nach $\S 46$ Abs. 1 S.1 StGB die Schuld des Täters. Eine gerechte Strafzumessung steht in einem proportionalen Verhältnis zur Schwere der Tat und der Schuld des Täters ${ }^{110}$ und gebietet, auf vergleichbare Sachverhalte unter Ansehung der Besonderheiten des Einzelfalls relativ gleich zu reagieren. ${ }^{111}$ Die Kronzeugenregelung widerspricht diesen Grundsätzen und ist zudem mit der Gefahr verbunden, dass diejenigen Beschuldigten, die von ihrem Schweigerecht Gebrauch machen, eine informelle Strafschärfung erhalten, da sie im Unterschied zum Kronzeugen nicht zur Aufklärung der Taten beigetragen haben. ${ }^{112}$ Dies würde das Recht des Angeklagten, sich nicht selbst belasten zu müssen, in Verfahren mit Kronzeugen zu einer Obliegenheit zum Geständnis relativieren. Die Kronzeugenregelung ist mit dem Schuldprinzip nicht zu vereinbaren. Es handelt sich bei ihr um eine Strafmilderung »an der Schuld des Täters vorbei. « ${ }^{113}$

107 Laut Mühlhoff / Pfeiffer, ZRP 2000, 121 (123), gibt es bislang keine statistischen Angaben zu der Frage, »in wie vielen Strafverfahren belastende Kronzeugenaussagen eine Schlüsselrolle gespielt haben (...).« Auch der seitdem veröffentlichten Literatur konnten solche Angaben nicht entnommen werden.

108 Exemplarisch Mühlhoff / Pfeiffer, ZRP 2000, 121 (125): »Rechtfertigungsgrund für eine solche Regelung ist indes das Interesse am Erhalt der Strafrechtspflege als ein prinzipiell taugliches Instrument zur Devianzkontrolle auf dem Gebiet schwerer und umfänglicher Kriminalität.«

109 Grundlegend: Hassemer in: Lüderssen (Hrsg.), V-Leute - Die Fall im Rechtsstaat, 1985, S.71 ff.; Jung, GA 2003, 191 ff., aus neuster Zeit mit anderer Gewichtung: Landau, NStZ 2007, $121 \mathrm{ff}$.

110 BVerfGE 6, 389 (439); 34, 261 (267); 75, 1 (16).

111 Jung, JZ 2004, 1155, 1157 f.; Schlüchter, ZRP 199765 (69); grundlegend: Streng, Strafzumessung und relative Gerechtigkeit, 1984.

112 Hassemer, StV 1986, 550, 552 f.; Ders., StV 1989, 80; Kempf, StV 1999, 67 (68); Weigend in: Vogler (Hrsg.), FS für Jescheck, S.1335; zur gleichen Problematik beim »Deal«: Prantl, Kursbuch 166 (2007), S.6 (9).

113 Streng, Strafrechtliche Sanktionen, 2. A. 2002, Rn. 470. Im Ergebnis auch: Fezer in: Eser u.a. (Hrsg.), FS für Lenckner, 1998, S.695; SK / Horn, § 46 Rn. 148; Jescheck / Weigend, Strafrecht AT, S.899; Koumbarakis, a.a.O., S.179; Montag, DRiZ 2004, 103; Volk, NJW 1996, 879 (881); Weigend, ZStW 109 (1997, 103 (113); vgl. auch: Kühne, Strafprozessrecht, Rn. 801, der den Strafnachlass jedoch (nur) als ein »Problem der Rechtspolitik und Rechtsethik « versteht. 


\section{Schlussfolgerung}

Die Kronzeugenregelung ist grundsätzlichen normativen Bedenken ausgesetzt und hat sich auch in ihrer praktischen Anwendung weder in Deutschland noch im Ausland bewährt. Sie wird dem Ziel einer jeden vernünftigen Kriminalpolitik, materielle Gerechtigkeit und eine effektive Strafrechtspflege unter Einhaltung eines fairen Verfahrens zu einer Synthese zu verbinden, nicht gerecht. Das Legitimationsdefizit der Kronzeugenregelung droht sich durch die Pläne der Bundesregierung gegenüber den bisherigen Regelungen sogar noch zu verschärfen, da ihr Anwendungsbereich auf den Bereich der allgemeinen Kriminalität ausgeweitet wird, die Kronzeugenregelung somit ihren ultima ratio-Charakter aufgibt und sich auf Kriminalitätsbereiche erstreckt, in denen die Taten mit herkömmlicher kriminalistischer Arbeit ohne weiteres aufgeklärt werden können. Nicht vergessen werden sollte, dass das Legitimationsdefizit auf das Ansehen der Justiz durchschlägt. Eine auf das Vertrauen der Bevölkerung angewiesene Strafrechtspflege sollte nicht den Eindruck aufkommen lassen, auf Kosten Dritter einen Tauschhandel mit der Gerechtigkeit zu betreiben. ${ }^{114}$ In nicht selten Fällen werden Kronzeugen von der Verteidigung in der Hauptverhandlung öffentlich »zerpflückt«. »Solche Zeugen stiften für die Anklage mehr Schaden als Nutzen«. ${ }^{115}$

114 Buttel, a.a.O., S.287; Dahs, NJW 1995, 553 (557); Lammer, JZ 1992, 510 (511); Lisken, Rechtsstaat - was sonst?, S.69; Mushoff, Forum Recht 2006, 80; Neumann, StV 1994, 273 (276); Roxin, Strafverfahrensrecht, S.90; Scheerer, StV 1989, 72 (83); Streng, Strafrechtliche Sanktionen, Rn. 470; Volk, NJW 1996, 879 (881).

115 Weigend in: Vogler (Hrsg.), FS für Jescheck, S.1340. 